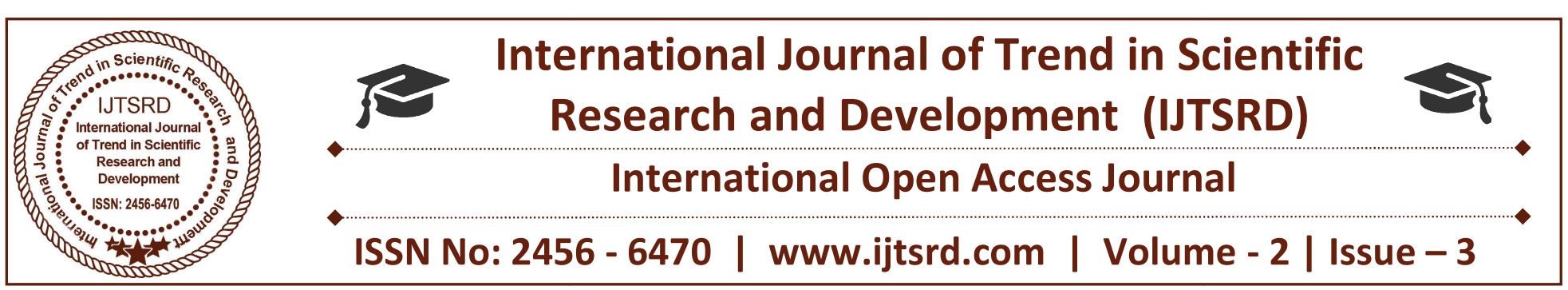

\title{
Smart Polyhouse Farming using IoT Environment
}

\author{
Raja. G \\ Assistant Professor, Department of Mechatronics \\ Engineering, Paavai Engineering College, \\ Salem, Tamil Nadu, India
}

\author{
Rajarathinam. D. R. P \\ Associate Professor, Department of Mechatronics \\ Engineering, Paavai Engineering College, \\ Salem, Tamil Nadu, India
}

\section{Abhiraj. R, Arunkrishnan, Febin Malik, Jesu Jorof Divin. J \\ Department of Mechatronics Engineering, Paavai Engineering College, Salem, Tamil Nadu, India}

\section{ABSTRACT}

A Polyhouse is a building where plants are grown. Polyhouses are often used for growing flowers, vegetables, fruits, and tobacco plant. Basic factors affecting plant growth are sunlight, water content in soil, temperature, etc. These physical factors are hard to control manually inside a Polyhouse and a need for automated design arises. Automatically controlling all the factors that affect plant growth is also a difficult task as it is expensive and some physical factors are inter-related, for example, temperature and humidity are related in a way when temperature raises humidity reduces therefore controlling both together is difficult. Because the temperature and humidity of Polyhouse must be constantly monitored to ensure optimal conditions, a wireless sensor network can be used to gather the data from point to point. A graphical user interface (GUI) is unified for the ease of operations by the farming community. System also allows transmission of process parameters, including emergency alarm signals via e-mail client server or alternatively sending a SMS on a mobile phone. A conventional chat has also been integrated with the GUI to add vibrancy to inter-user communication. This feature can be embedded in upcoming $3 \mathrm{G}$ mobile technology. Simulations and video tutorials can also be integrated in the web server for teaching the farming community. Such integrated approach greatly widens the socio-economic possibilities for farmers through interaction with modern technological resources.

Keywords: Graphical user interface, Polyhouse

\section{INTRODUCTION}

In human activity, telecommunication and internet play very important role in day to day life. In modern society, no other technology has made such impact on communication. Web browser services form one of the core foundations of a successful information technology. Internet is information sharing technology. It not only sell product but also improves design engineering system, manufacture and test final product. Thus, to improve overall quality the balanced and justified usage of internet facilities is very important for reduction of design cycles. Last few decade, growth of mobile technology and internet increased exponentially. India is the large developing country. Here, internet services have been proven to be one of the most efficient systems to couple with mobile telephony. Mechanization and modernization of agriculture make considerable impact to infuse these two technologies. In India two-third of population is depend on agriculture. Agriculture uses $85 \%$ of available fresh water resources worldwide. Because of population growth and increased food demand, this percentage will continue to be dominant in water consumption. 
In last decade, ground water level has been decreasing day by day due to unplanned use of water and lack of rains. Irrigation system improves the yield and quality of crops. Also, saves huge amount of water. In present micro irrigation system such as drip and Sprinkler irrigation, water being provided directly to root zone of plants and this results in water saving and labor work savings. Indian farmers face several challenges such as availability of water, small land holding, poor yield due improper methods of farming, natural phenomena such as rainfall and lack of knowledge of modern methods of agriculture. Recently, for low cost production new intelligent systems are being developed in agricultural. To growing multiple crops and for making efficient use of land and resources, plant factory provide high yield of crop in agriculture. Main source for Plant growth is that surrounding climates such as humidity, temperature, $\mathrm{CO} 2$ and light intensity and these factors are very important to be monitored and maintained to increase yield of crop. The wireless sensors networks (WSN) perform a specific task to defined as the collection of sensor nodes that and they are representing one of the technological solutions to automates and improve the management of crops. Poly house farming is an alternative new technique in agriculture gaining foothold in rural India and can be successfully employed for niche areas of agriculture. A typical Poly house (metal structure covered with Polythene) is from $400-10,000 \mathrm{~m} 2$; this makes them suitable for farmers with small land holding also. A low - to medium - cost Poly house could cost between Rs. 125 to Rs.500 per square meter in India, whereas a highcost, fully-automated Poly house costs Rs. 2,000 per square meter. Most Indian farmers cannot afford such high costs but group of farmers and co-operatives can use such systems.

Farmers do require expert guidance to use this new technology of Poly house farming. This methodology of farming reduces dependency on rainfall and makes the optimum use of land and water resources; typical gains may be three times those of traditional farming. Poly house farming/ shelter farming is an alternative new technique in agriculture gaining foothold in rural India. It reduces dependency on rainfall and makes the optimum use of land and water resources. Potentially, poly house farming can help the farmer generate income round the year growing multiple crops. This also helps them spread their risks.

Poly house farming enables cultivation of crops that can give maximum yield on specific days (e.g. roses on Valentine's day) and exotic crops that can't be normally grown in Indian conditions. It also enables cultivation of regular crops off-season, thus fetching the farmer a higher price (e.g. tomato, chilly, capsicum, brinjal, cucumber, cabbage, cauliflower). Apart from this cut Flowers can be grown round the year. Poly house farming entails construction of a metal structure covered by polythene. Parameters such as moisture, soil nutrients and temperature in the poly house are controlled to ensure timely and abundant yields.

Poly house farming process requires expertise in three areas - construction of the structure, cultivation techniques and marketing. Within cultivation, the preharvest techniques include irrigation, providing fertilizer's, pesticides and micro-nutrients, maintaining temperature, humidity and sunlight in the poly house, cutting, pruning and cleaning practices and controlling $\mathrm{pH}$ and electrical conductivity of the soil. The post-harvest techniques include cutting, storage cooling chambers and transport by cooling vans. The government as well as private poly house construction companies provide practical training to the farmers for a month or two. However, this short training is not sufficient to understand the complex poly house farming techniques, particularly the preharvest techniques.

\section{NE SYSTEM ANALYSIS}

\section{A. Block Diagram of Proposed System}

All sensor data are collected in database and monitored on PC as well as web browser. Temperature and Humidity will be controlled using exhaust fan. When temperature and humidity is high then exhaust fan is on otherwise off. Then another important part is a carbon dioxide. Soil moisture sensor measures the moisture of soil, if moisture is dry and water tank level is high then water will be supplied on root zone of the crop. But, if soil moisture is dry and water level is low then pump will be off. In the proposed method, the greenhouse controller senses the change in temperature and relative humidity with the help of input sensors and process the output to take appropriate control action. The proposed system is a low cost and user friendly system with high stability and reliability. 


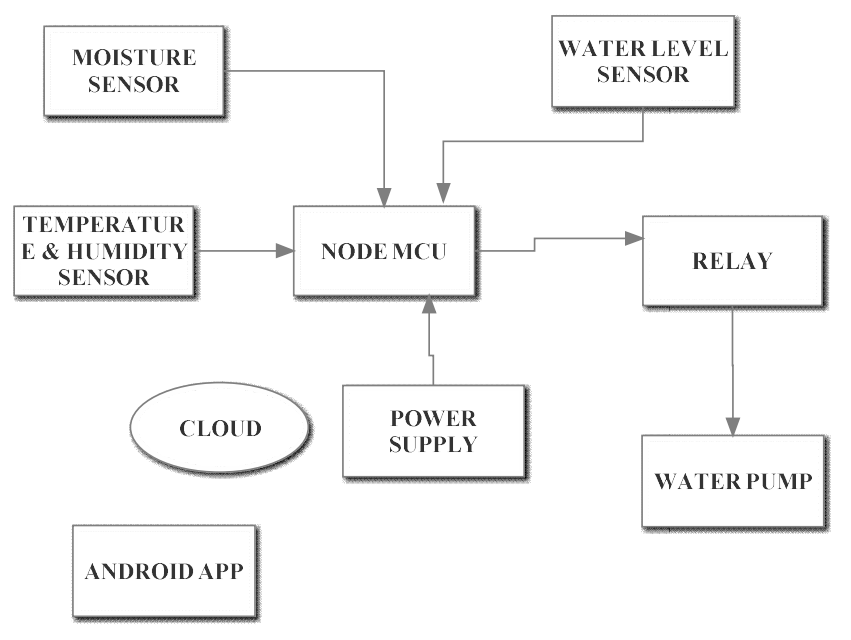

Fig. 1: Block Diagram of Proposed System.

Internet of Things (IoT) is the network of physical things embedded with electronic circuits, sensors, software and network connection which enables these things to exchange data from one another. IoT is the fusion of the digital and physical world. In a world of IoT, millions of things or devices will be interconnected and uniquely identified on the Internet. The Internet of Things allows objects to be sensed and controlled remotely across existing network infrastructure, creating opportunities for more direct integration between the physical world and computerbased systems, and resulting in improved efficiency, accuracy and economic benefit.

In near future, IoT is expected to provide many more services like advanced connectivity of physical objects over a wide network and also many applications. Node-MCU is an open source IoT platform. It includes firmware which runs on the ESP8266 Wi-Fi SoC from Es press if Systems, and hardware which is based on the ESP-12 module. The term "Node-MCU" by default refers to the firmware rather than the dev kits. The firmware uses the Lua scripting language. It is based on the eLua project, and built on the Es press if Non-OS SDK for ESP8266. It uses many open source projects, such as luacjson, and spiffs. The Node-MCU (Node Micro-Controller Unit) is an open source software and hardware development environment that is built around a very inexpensive System-on-a-Chip (SoC) called the ESP8266. The ESP8266, designed and manufactured by Es press if Systems, contains all crucial elements of the modern computer: CPU, RAM, networking (wi-fi), and even a modern operating system and SDK. When purchased at bulk, the ESP8266 chip costs only \$2 USD a piece.
That makes it an excellent choice for IoT projects of all kinds.

However, as a chip, the ESP8266 is also hard to access and use. You have to solder wires, with the appropriate analog voltage, to its PINs for the simplest tasks such as powering it on or sending a keystroke to the "computer" on the chip. And, you have to program it in low-level machine instructions that can be interpreted by the chip hardware. While this level of integration is not a problem when the ESP8266 is used as an embedded controller chip in mass-produced electronics, it is a huge burden for hobbyists, hackers, or students who want to experiment with it in their own IoT projects.

\section{Power Supply}

The power supplies are designed to convert high voltage $\mathrm{AC}$ mains electricity to a suitable low voltage supply for electronic circuits and other devices. A RPS (Regulated Power Supply) is the Power Supply with Rectification, Filtering and Regulation being done on the $\mathrm{AC}$ mains to get a Regulated power supply for Microcontroller and for the other devices being interfaced to it. A power supply can by broken down into a series of blocks, each of which performs a particular function. A d.c power supply which maintains the output voltage constant irrespective of a.c mains fluctuations or load variations is known as "Regulated D.C Power Supply".

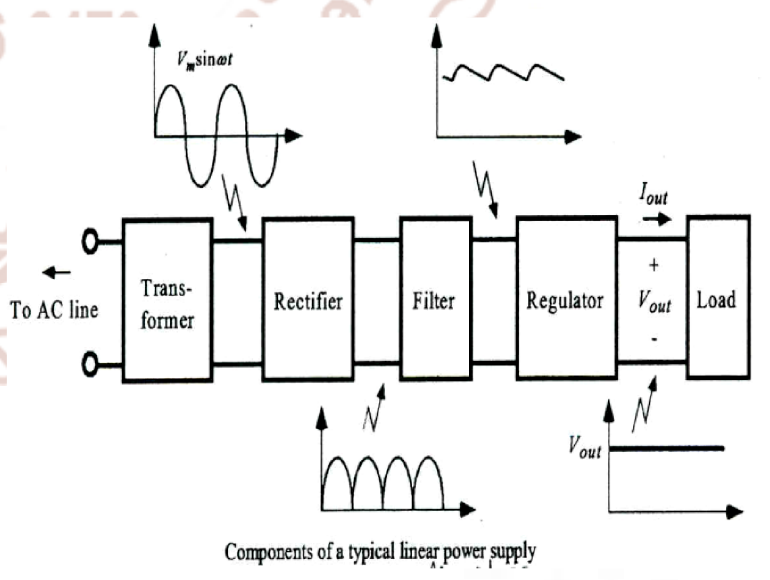

Fig 2: Regulated Power Supply System

\section{RELAY}

A simple electromagnetic relay, such as the one taken from a car in the first picture, is an adaptation of an electromagnet. It consists of a coil of wire surrounding a soft iron core, an iron yoke, which provides a low reluctance path for magnetic flux, a moveable iron armature, and a set, or sets, of contacts; 
two in the relay pictured. The armature is hinged to the yoke and mechanically linked to a moving contact or contacts. It is held in place by a spring so that when the relay is de-energized there is an air gap in the magnetic circuit. In this condition, one of the two sets of contacts in the relay pictured is closed, and the other set is open. Other relays may have more or fewer sets of contacts depending on their function. The relay in the picture also has a wire connecting the armature to the yoke. This ensures continuity of the circuit between the moving contacts on the armature, and the circuit track on the Printed Circuit Board (PCB) via the yoke, which is soldered to the PCB.

\section{Water Level Sensor}

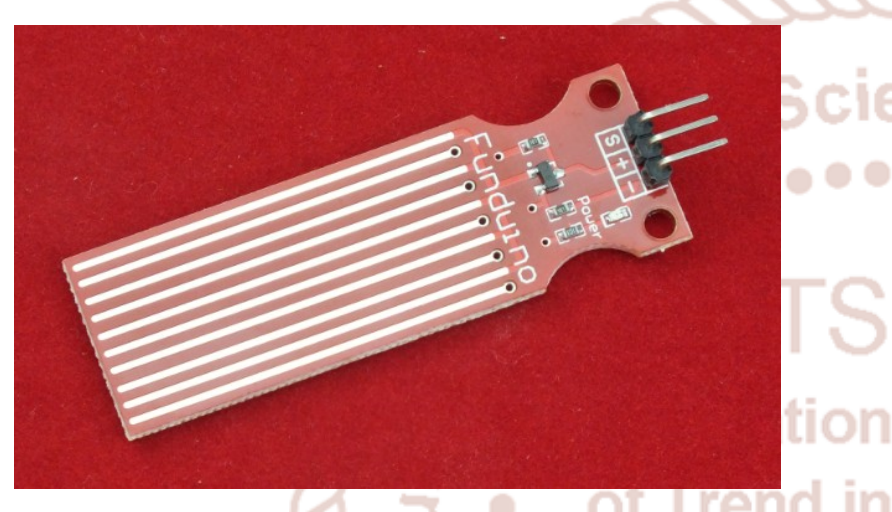

Fig 3: Diagram of Water Level Sensor

The sensing probe element consists of a special wire cable which is capable of accurately sensing the surface level of nearly any fluid, including water, salt water, and oils. The sensor element is electrically insulated and isolated from the liquid into which it is inserted, and will not corrode over time. Unlike, other sensors, the measurement range is adjustable from a few centimeters to over several meters.

The reading is reported back as an analog voltage ranging from $0 \mathrm{~V}$ to $3 \mathrm{~V}$ where $0 \mathrm{~V}$ represents the sensor not being submersed, and $3 \mathrm{~V}$ represents the maximum water level. The water level sensor can be easily calibrated to nearly any range and any fluid in the field with the use of two buttons. One button records the minimum fluid level (0V level), and the the other button records the maximum fluid level $(3 \mathrm{~V}$ level). After being calibrated, the sensor will return a value of 0 to 3 volts linear with the liquid level.

The water level sensor is compact, low cost, low power, easy to use, and can take an accurate reading in less than a second. The sensor has an easy to mount water resistant enclosure. The cable can be made taut with a cable clamp, which can be mounted to the side of a reservoir, to a small weight, or to a stick.

\section{Humidity Sensor}

Humidity is the presence of water in air. The amount of water vapor in air can affect human comfort as well as many manufacturing processes in industries. The presence of water vapor also influences various physical, chemical, and biological processes. Humidity measurement in industries is critical because it may affect the business cost of the product and the health and safety of the personnel. Hence, humidity sensing is very important, especially in the control systems for industrial processes and human comfort.

In agriculture, measurement of humidity is important for plantation protection (dew prevention), soil moisture monitoring, etc. For domestic applications, humidity control is required for living environment in buildings, cooking control for microwave ovens, etc. In all such applications and many others, humidity sensors are employed to provide an indication of the moisture levels in the environment.

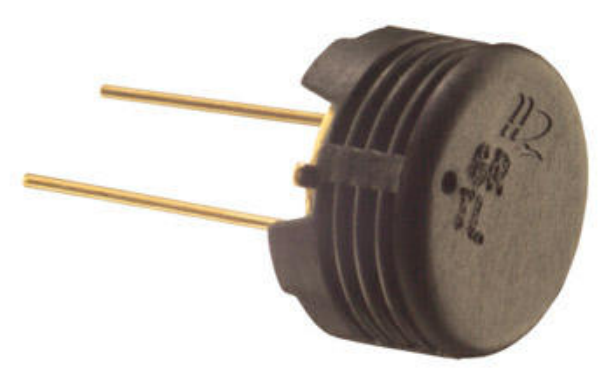

Fig. 4. Diagram of Humidity Sensor

\section{Moisture Sensor}

The Moisture sensor is used to measure the water content(moisture) of soil. when the soil is having water shortage, the module output is at high level, else the output is at low level. This sensor reminds the user to water their plants and also monitors the moisture content of soil. It has been widely used in agriculture, land irrigation and botanical gardening. The Soil Moisture Sensor uses capacitance to measure dielectric permittivity of the surrounding medium.

In soil, dielectric permittivity is a function of the water content. The sensor creates a voltage proportional to the dielectric permittivity, and therefore the water content of the soil. The sensor 
averages the water content over the entire length of the sensor. There is a $2 \mathrm{~cm}$ zone of influence with respect to the flat surface of the sensor, but it has little or no sensitivity at the extreme edges. The Soil Moisture Sensor is used to measure the loss of moisture over time due to evaporation and plant uptake, evaluate optimum soil moisture contents for various species of plants, monitor soil moisture content to control irrigation in greenhouses and enhance bottle biology experiments.

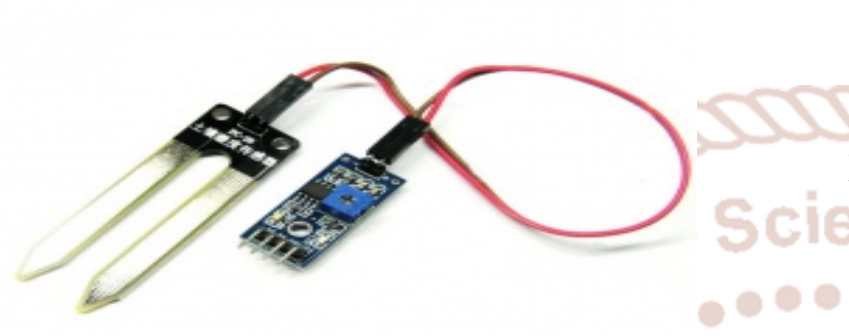

Fig.5. Diagram of Moisture Sensor

\section{Software Implementation}

By implementing automation inside the poly house all things are monitored through the mobile by IoT. Here the temperature sensor senses the temperature, humidity sensors sense the moisture. If the temperature goes down, the light will glow and the temperature increases. All things inside the farm are controlled by Arduino. This product can be implemented easily in the farm. Anyone can farm the poly house with few agricultural knowledges.

Android is a mobile operating system (OS) based on the Linux kernel and currently developed by Google. With a user interface based on direct manipulation, Android is designed primarily for touch screen mobile devices such as smart phones and tablet computers, with specialized user interfaces for televisions (Android TV), cars (Android Auto), and wrist watches (Android Wear). The OS uses touch inputs that loosely correspond to real-world actions, like swiping, tapping, pinching, and reverse pinching to manipulate on-screen objects, and a virtual keyboard. Despite being primarily designed for touch screen input, it also has been used in game consoles, digital cameras, regular PCs (e.g. the HP Slate 21) and other electronics.

\section{Embedded C}

Looking around, we find ourselves to be surrounded by various types of embedded system. Be it a digital camera or a mobile phone or a washing machine, all of them has some kind of processor functioning inside it. Associated with each processor is the embedded software. If hardware forms the body of an embedded system, embedded processor acts as the brain, and embedded software forms its soul. It is the embedded software which primarily governs the functioning of embedded systems.

\section{OOPS}

Object-oriented programming (OOP) is a programming paradigm based on the concept of "objects", which may contain data, in the form of fields, often known as attributes; and code, in the form of procedures, often known as methods. A feature of objects is that an object's procedures can access and often modify the data fields of the object with which they are associated (objects have a notion of "this" or "self"). In OOP, computer programs are designed by making them out of objects that interact with one another. There is significant diversity of OOP languages, but the most popular ones are classbased, meaning that objects are instances of classes, which typically also determine their type.

Many of the most widely used programming languages (such as $\mathrm{C}++$, Object Pascal, Java, Python etc.) are multi-paradigm programming languages that support object-oriented programming to a greater or lesser degree, typically in combination with imperative, procedural programming. Significant object-oriented languages include Java, C++, C\#, Python, PHP, Ruby, Perl, Object Pascal, Objective-C, Dart, Swift, Scala, Common Lisp, and Smalltalk.

\section{Features}

Object-oriented programming uses objects, but not all of the associated techniques and structures are supported directly in languages that claim to support OOP. The features listed below are, however, common among languages considered strongly classand object-oriented (or multi-paradigm with OOP support), with notable exceptions mentioned.

\section{Advantages}

- Less time delays

- Quick response time 
- Whole system is Fully automated

- Robust system, require low power

- Less water consumption

\section{CONCLUSION}

The usage of the sensors and IoT to generate control signals for the irrigation of the farm, there is a substantial saving of the resources. The system also relieves the farmer from being physically present in the farm to operate the irrigation system. The time saved can be used for managing the rest of the activities on the farm. The system also enables the farmer to manage a large farm with minimum manpower. This reduces the production cost of the produce. This in turn increases the profit earned by the farmer. The control system monitors the temperature, humidity, moisture and water level in the greenhouse to solve the problem of plant disease. To make up for this weak point, this system proposes and collects the information regarding temperature, humidity, soil moisture so that the change of condition of crops depending on internal environment factors of greenhouse can be estimated.

\section{REFERENCES}

1. D. E. Upasani, S. B. Shrote and V. P. Wani, FPGA implementation of intelligent climate control for greenhouse, International journal of Computer application, 1(18), 2010

2. G.K. Banerjee and Rahul Singhal, Microcontroller based Polyhouse Automation Controller, International Symposium on Electronic System Design, 2010

3. Rokade, Assistance and Control System for Polyhouse Plantation, M. Des. Thesis, IDC IIT Bombay, 2004.

4. T. Ahonen, R. Virrankoski, M. Elmusrati, "Greenhouse Monitoring with Wireless Sensor Network", IEEE /ASME International Conference on Mechatronic and Embedded Systems and Applications, 2008

5. Dae-Heon P. and Jang-Woo P., 2011. Wireless sensor network-based greenhouse environment monitoring and automatic control system for dew condensation prevention. Journal of Sensors, Vol. 11 , pp. $3640-3651$.

6. Dussion M. F., 1989. Greenhouse and energy. French Agency for Energy Management, p. 96.
7. Eddahhak A., 2009. Development of a system for monitoring the climate and managing the drip fertilizing irrigation in greenhouse by using LabVIEW software. National PhD, Faculty of Sciences, Meknes, Moulay Ismail University, Morocco

8. Eddahhak A., Lachhab A., Ezzine L. and Bouchikhi B., 2007. Performance evaluation of a developing greenhouse climate control with a computer system. AMSE Journal Modelling C, Vol. 68, No. 1, pp. 53-64.

9. El-Fadl A., El Kherrak H., Claustriaux J. et Mounhim H., 1996. Computer aided management of greenhouse climate and influence on the culture of melon in the region of Souss. Choukr-Allah R. (ed.). Protected cultivation in the Mediterranean region. Notebooks Options Mediterranean Vol. 31, pp. 99-108.

10. El Harzli M., 2009. Study and realization of a multifunctional sensor, heat flux, temperature and humidity. Application to the greenhouse control. National PhD , Faculty of Sciences, Meknes, Moulay Ismail University, Morocco.

11. Hayat Khiyal M. S., Khan A. and Shehzadi E., 2009. SMS Based Wireless Home Appliance Control System (HACS) for Automating Appliances and Security. Issues in Informing Science and Information Technology, Vol. 6, pp. 887-894.

12. Lajara R., Alberola J. and Pelegrí-Sebastiá J., 2011. A Solar Energy Powered Autonomous Wireless Actuator Node for Irrigation Systems. Journal of Sensors. Vol. 11, pp. 329-340.

13. Laszewski, G., Younge, A., He, X., Kunze, M., Tao, J., Fu, C., and Wang, L. Cloud computing: a perspective study. New Generation Computing, 28(2):137-146.

14. Li X. H., Cheng X., Yan K. and Gong P., 2010. A monitoring system for vegetable greenhouses based on a wireless sensor network. Journal of Sensors, Vol. 10, pp. 8963-8980.

15. Ji-chun Zhao; Ju-feng Zhang; Yu Feng; Jian-xin Guo, "The study and application of the IOT technology in agriculture," Computer Science and Information Technology (ICCSIT), 2010 3rd IEEE International Conference on, vol.2, no., pp.462,465, 9-11 July 2010. 
International Journal of Trend in Scientific Research and Development (IJTSRD) ISSN: 2456-6470

16. J. W. Overstreet and A. Tzes, "An Internet-based real-time controlengineering laboratory", IEEE Control Syst., Vol. 19, pp. 19-33, 1999.

17. P. L. Regtien, M. Halaj, E. Kurekova and P. Gabko, "COMET: A multimedia internet based platform for education in measurement", Measurements, Vol. 40, No. 2, pp. 171-182, 2007. TRAI's press release. (Referred October 2009). [Online]. Available: http://www.trai.gov.in.
18. Agricultural Statistics at a Glance (2008), Department of Agriculture and Cooperation, Ministry of Agriculture, Government of India.

19. S. K. Sanwal, K. K. Patel and D. S. Yadav, "Vegetable Production under Protected Conditions in NEH Region", ENVIS Bulletin: Himalayan Ecology, Vol. 12, No. 2, 2004.

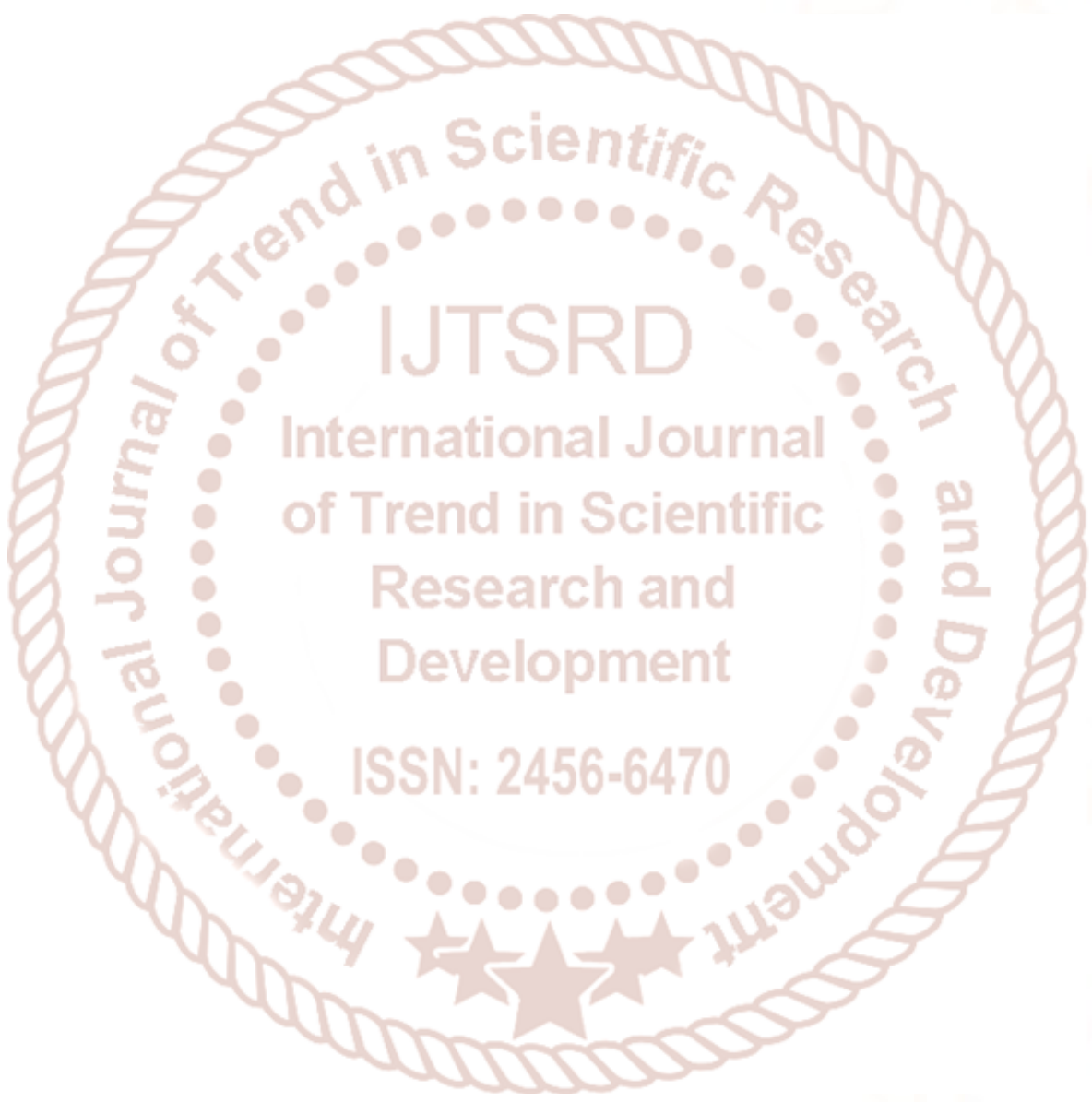

